\title{
Insulin-like growth factor 2 (IGF2) and IGF-binding protein 1 (IGFBP1) gene variants are associated with overfeeding-induced metabolic changes
}

\author{
O.Ukkola ${ }^{1,2}$, G.Sun ${ }^{3}$, C. Bouchard ${ }^{1}$ \\ ${ }^{1}$ Pennington Biomedical Research Center, Louisiana State University, Baton Rouge, La, USA \\ ${ }^{2}$ Department of Internal Medicine and Biocenter Oulu, University of Oulu, Oulu, Finland \\ ${ }^{3}$ Discipline of Medical Genetics, Memorial University of Newfoundland, St. John's, Newfoundland, Canada
}

\section{Abstract}

Aims/hypothesis. The aim of this study was to investigate the role of insulin-like growth factor 1 (IGF1), $I G F 2, I G F$ binding protein $1(I G F B P 1)$ and $I G F B P 3$ gene variants on the metabolic changes observed in response to a 100-day overfeeding protocol conducted with 12 pairs of monozygotic twins.

Methods. Genotyping was done by PCR-RFLP and DNA sequencer methods. Body fat measurements included hydrodensitometry and abdominal fat from computed tomography. Plasma glucose and insulin during fasting and in response to an OGTT were assayed. Plasma lipids were measured enzymatically.

Results. In response to caloric surplus, fasting plasma insulin $(p<0.05)$ and OGTT insulin $(p=0.004)$ but not glucose area, increased more among the subjects with IGF2 Apa I GG $(n=12)$ than those with $\mathrm{AA}+\mathrm{AG}(n=12)$. The changes were independent of changes in total fatness. The subjects with IGFBPI $B g l$ II $A A(n=8)$ showed greater increases in abdom- inal visceral fat $(p<0.01)$, OGTT insulin area $(p=0.05)$ and total cholesterol $(p<0.03)$ with overfeeding than the subjects with AG + GG $(n=16)$. $I G F B P 3$ Nde $I$ and the IGF1 (CT) markers were not associated with responsiveness to overfeeding. Conclusion/interpretation. Insulin sensitivity decreased in the subjects with IGF2 Apa I GG and the subjects with IGFBP1 Bgl II AA showed an accumulation of abdominal visceral fat and the early symptoms of the metabolic syndrome after long-term caloric surplus. Genetic variation at the $I G F 2$ and $I G$ FBP1 loci could be among the factors responsible for the inter-individual differences observed in the response to long-term alterations in energy balance and should be further investigated in larger cohorts. [Diabetologia (2001) 44: 2231-2236]

Keywords IGF1, IGF2, IGFBP1, IGFBP3, gene, polymorphism, insulin, abdominal fat, lipids, overfeeding.
Insulin-like growth factors 1 and 2 (IGF1 and IGF2) are important for regulating growth and metabolism [1]. In biological fluids, IGFs are normally bound to specific binding proteins, insulin-like growth factor

Received: 26 March 2001 and in revised form: 31 August 2001

Corresponding author: C. Bouchard, Pennington Biomedical Research Center, Louisiana State University, 6400 Perkins Road, Baton Rouge, LA 70808-4124, USA, e-mail: bouchac@ pbrc.edu

Abbreviations: IGFBP, Insulin-like growth factor binding protein; IGF, insulin-like growth factor; CT, computed tomography binding proteins, IGFBPs [2]. IGFs exert several insulinomimetic effects such as reducing plasma concentrations of insulin and glucagon and stimulating lipid oxidation [3]. An inverse association between total $I G F 1$ concentrations and visceral adipose depot has been reported $[4,5]$. Thus the IGF and IGFBP genes could be involved in insulin resistance and other obesity-related phenotypes as well as in the responsiveness to dietary changes. A microsatellite polymorphism at the $I G F 1$ gene showed both linkage and association with body composition phenotypes in the sedentary state and in response to endurance training [6]. In addition, an IGF2 gene variant has been shown to affect body weight variability and 
IGF2 concentrations [7]. The aim of this study was to assess whether genetic variations at the IGF1,IGF2, $I G F B P 1$ and IGFBP3 gene loci explained the observed large inter-individual variations in the morphological and metabolic changes [8] brought about by a long-term (100 days) overfeeding protocol carried out on 12 pairs of monozygotic twins.

\section{Subjects and methods}

Twelve pairs of young (aged $21 \pm 2$ years) male identical twins provided written consent to participate in an overfeeding study approved by the Laval University Medical Ethics Committee and the Office for the Protection from Research Risks of the National Institutes of Health (Bethesda, Md., USA). The men were housed in a closed section of a dormitory on the campus of Laval University. During the overfeeding period the men ate a $4.2 \mathrm{MJ}$ per day (1000 kcal) energy surplus, six days a week, over 100 days. The subjects were under 24-h supervision by members of the project staff who lived with them. The specific aims, study design, and methodology of this overfeeding protocol have been described [8]. In this study, the twins are considered as 24 subjects and as 12 pairs. Each man stayed in the unit for 120 consecutive days: 14 days for the assessment of baseline daily energy intake, 3 days for testing before the period of overfeeding, 100 days for the period of overfeeding, and 3 days for testing after the period of overfeeding.

Body composition and regional fat distribution measurements. The data on body weight represent the mean measurements of 3 days, the day on which the underwater weighing was done and the days before and after. Body density was analysed by the hydrostatic weighing technique [9] and fat mass was calculated with a standard equation [10]. Abdominal computed tomography (CT) scanning was done before and after the overfeeding period with a Siemens Somatom DRH scanner (Erlangen, Germany) [11].

Plasma glucose, insulin and glucagon measurements. The plasma glucose concentration was measured enzymatically [12], and the plasma insulin by radioimmunoassay (RIA) [13]. Glucagon was analysed in unextracted plasma by radioimmunoassay with the $30 \mathrm{~K}$ antibody [14]. Glucose and insulin total areas under the curve during the OGTT were assessed with the trapezoid method.

Plasma lipid measurements. Total plasma cholesterol was analysed using the enzymatic kit CHOD-PAP from Boehringer (Mannheim, Germany). High-density lipoprotein cholesterol was assayed with the same method after separation of HDL from low-density and very-low-density lipoproteins. Plasma triglycerides were analysed enzymatically with the A-Gent kit (Abbott Laboratories, South Pasadena, Calif., USA).

Plasma testosterone and IGF1 analysis. The testosterone concentrations were measured by RIA [15]. IGFI concentrations were determined with the commercial RIA SM-C kit from Nichols Institute Diagnostics (San Juan Capistrano, Calif., USA). The samples were fasting plasma and all the assays were done shortly after the overfeeding protocol was completed.

Genotype measurements. Genomic DNA was isolated from lymphoblastoid cell lines [16]. IGF1 (CT) $)_{\mathrm{n}}$ polymorphism and
PCR and DNA sequencer techniques by which it was uncovered have been described [6]. The primer sequences were as follows: forward primer, 5'-TTG-TGT-CAA-CTG-CTGATA-TG-3'; reverse primer, 5'-AAC-CAA-AAC-ATC-ATTCCC-TA-3'. The IGF2 Apa I polymorphic site is located in the 3' untranslated region at the IGF2 gene. The primers were derived from previously published data [17]: forward primer, 5'-CTT-GGA-CTT-TGA-GTC-AAA-TTG-G-3'; reverse primer, 5'-CCT-CCT-TTG-GTC-TTA-CTG-GG-3'. The amplification product was digested at $25^{\circ} \mathrm{C}$ for $16 \mathrm{~h}$ with $10 \mathrm{U}$ of Apa I (BRL) enzyme. The fragments were separated on a $2 \%$ agarose gel and visualized under UV light after staining with ethidium bromide. The $B g l I I$ restriction site is in the first intron of the IGFBP1 gene and PCR analysis of the polymorphism was carried out in a volume of $15 \mu$ l containing $100 \mathrm{ng}$ DNA, $0.3 \mu \mathrm{mol} / 1$ of each primer, $0.2 \mathrm{mmol} / 1$ of each of the dNTPs, 0.5 unit Taq polymerase in a standard buffer and Q solution (Qiagen, Mississauga, Ontario, Canada). The primers for the IGFBPI Bgl II polymorphism were as follows: forward, 5'-CAC-GTA-GTT-GGG-GAA-GGA-GC-3'; reverse, 5'GGC-AAT-GAA-TGG-AAG-TGA-AGC-3'. The PCR was started at $94^{\circ} \mathrm{C}$ for $5 \mathrm{~min}, 55^{\circ} \mathrm{C}$ and $72^{\circ} \mathrm{C}$ for $1 \mathrm{~min}$ followed by 30 cycles at $94^{\circ} \mathrm{C}, 55^{\circ} \mathrm{C}$ and $72^{\circ} \mathrm{C}$ for $30 \mathrm{~s}$ and 1 cycle at $72^{\circ} \mathrm{C}$ for 10 min using a thermal cycler (model 9600: Perkin-Elmer Cetus Instruments, Branchburg, N.J., USA). The amplified product was digested at $37^{\circ} \mathrm{C}$ for $18 \mathrm{~h}$ with 20 units of $B g l$ II. The fragments were separated on a $2.5 \%$ agarose gel. The Nde I restriction site is in the third intron of the IGFBP3 gene and genotyping of this polymorphism was done as described earlier [18]. The primer sequences were as follows: forward primer, 5'-CTC-CGA-GTC-ACT-GGC-ATT-TC-3'; reverse primer, 5'-ACC-AGC-CCT-TGT-AGA-ACC-TC-3'.

Statistical analysis. The differences in absolute changes between genotypes at a given marker were assessed by a Student's $t$ test. The 24 subjects considered as unrelated persons and the phenotype mean of each of the 12 pairs were analysed. Statistical analyses were carried out with the SAS statistical package (SAS institute, Cary, N.C., USA). A $p$ value of less than 0.05 was considered statistically significant.

\section{Results}

The effects of overfeeding on measures of body composition in this sample of identical twins have been reported [8]. In this study, the IGF1 dinucleotide $(\mathrm{CT})_{\mathrm{n}}$ polymorphism showed six alleles, the 189/189 bp homozygotes (189/189 bp) being the most common genotype $(n=14)$. Because of the low number of rare allele homozygotes, all other genotypes $(n=10)$ were pooled together (non-189/189 bp) for the purpose of comparison. Thus, IGF2 Apa I A allele homozygotes $(n=2)$ were pooled together with AG heterozygotes $(n=10)(\mathrm{AA}+\mathrm{AG})$ and compared with $\mathrm{G}$ allele homozygotes $(n=12)(\mathrm{GG})$. In addition, IGFBPI $B g l$ II $\mathrm{G}$ allele homozygotes $(n=2)$ were pooled together with GA heterozygotes $(n=14) \quad(\mathrm{GG}+\mathrm{GA}) .189 / 189$ bp subjects of the IGF1 (CT) n polymorphism had higher IGF1 concentrations before [1144.5 (288.0); $p=0.019]$ and after [1195.9 (419.6); $p=0.011]$ overfeeding than subjects who were non-189/189 bp [before: 801.3 (374.4) and 
Table 1. Effect of overfeeding on obesity-related phenotypes in male twins in relation to the IGF2 APA I and IGFBPI BGL II genotypes

\begin{tabular}{|c|c|c|c|c|}
\hline & \multicolumn{2}{|c|}{ IGF2 APA I genotypes } & \multicolumn{2}{|c|}{ IGFBP1 BGL II genotypes } \\
\hline & $\mathrm{AA} / \mathrm{AG}(n=12)$ & $\mathrm{GG}(n=12)$ & GG/GA $(n=16)$ & $\mathrm{AA}(n=8)$ \\
\hline \multicolumn{5}{|c|}{ Body weight (kg) } \\
\hline After & $67.8(2.3)$ & $69.1(2.5)$ & $69.1(2.3)$ & $67.2(2.4)$ \\
\hline Change & $7.9(0.7)$ & $8.4(0.7)$ & $7.7(2.2)$ & $8.9(2.7)$ \\
\hline \multicolumn{5}{|c|}{ Fat mass (kg) } \\
\hline Change & $4.7(0.6)$ & $6.1(0.5)$ & $5.4(0.4)$ & $5.4(0.9)$ \\
\hline \multicolumn{5}{|c|}{ Subcutaneous fat (mm) } \\
\hline Before & $69.1(3.9)$ & $82.8(7.4)$ & $79.1(6.0)$ & $69.6(4.4)$ \\
\hline After & $114.9(7.2)$ & $144.0(9.9)^{* *}$ & $130.3(8.1)$ & $127.6(12.6)$ \\
\hline Change & $45.8(5.7)$ & $61.2(4.2)^{*}$ & $51.2(3.5)$ & $58.0(9.4)$ \\
\hline \multicolumn{5}{|c|}{ Abdominal visceral fat $\left(\mathrm{cm}^{2}\right)$} \\
\hline Before & $4.56(0.24)$ & $4.43(0.23)$ & $4.63(0.20)$ & $4.20(0.27)$ \\
\hline After & $4.77(0.17)$ & $5.07(0.45)$ & $4.79(0.23)$ & $5.18(0.56)$ \\
\hline Change & $0.21(0.17)$ & $0.64(0.31)$ & $0.16(0.16)$ & $0.97(0.39) * *$ \\
\hline
\end{tabular}

Values are means (SE). Changes are post-overfeeding minus pre-overfeeding values. $p$ values based on 24 subjects considered as unrelated persons are as follows:

$* p<0.05$

$* * p \leq 0.03$

after 771.1 (288.8)]. However, the variant was not associated with any of the phenotype changes in response to overfeeding.

The influence of the long-term caloric surplus on obesity-related phenotypes and hormonal changes by IGF2 Apa I and IGFBPI Bgl II genotypes is shown (Tables 1, 2 and Fig.1). IGF2 Apa I GG subjects had higher fat mass before $(p<0.05)$ and after $(p<0.03)$ overfeeding as well as higher subcutaneous fat $(p<0.03)$, leptin $(p<0.05)$ but lower total testosterone concentrations $(p<0.01)$ after overfeeding compared with the AA/AG subjects. The IGF2 Apa $I$ GG subjects also experienced greater increases in the amount of subcutaneous fat $(p<0.05$; Table 1$)$, leptin $(p<0.05 ;$ Table 2$)$ and fasting insulin $(p<0.05$; Table 2$)$ in response to overfeeding. Furthermore, the OGTT insulin area increased more among subjects with the genotype GG ( $p=0.004$; Fig.1). The increase in OGTT insulin area in the IGF2 Apa I GG subjects remained significant after adjustment for the change in fat mass. However, the changes in OGTT glycaemic area did not differ by IGF2 Apa I genotype. In contrast, total $(p<0.01)$ and free testosterone (NS) showed less increase among subjects with the IGF2 Apa I GG. Finally, the IGF1 concentrations tended to decrease in GG but increase in AA/AG subjects (Table 2).

Homozygotes for the IGFBPI Bgl II $A$ allele had higher glucagon values both before $(p<0.03)$ and af-
$* * * p<0.01$ between genotype groups

$p$ values when phenotype means of each pair are compared $(n=12)$ are as follows:

$*$ and $* * \mathrm{NS}, * * * p=0.019$ for the difference in the change in abdominal visceral fat

ter overfeeding $(p<0.03)$ and a higher amount of abdominal visceral fat $(p<0.01)$ than homozygotes or heterozygotes for the $\mathrm{G}$ allele (Table 2 ). IGF1 concentrations were lower before $(p<0.05)$ and after $(p<0.03)$ the caloric surplus among the IGFBP1 Bgl II $A A$ homozygotes. In addition, IGFI concentrations tended to decrease among AA but increase in GG/GA subjects (Table 2). The IGFBP1 Bgl II A allele homozygotes showed greater increases in abdominal visceral fat $(p<0.01)$, OGTT insulin area and total cholesterol $(p<0.03)$ with overfeeding than $\mathrm{G}$ allele homozygotes or heterozygotes (Tables 1,2). Increases in OGTT insulin area and cholesterol in the IGFBPI Bgl II A allele homozygotes were not statistically significant after adjustment for the change in abdominal visceral fat (data not shown). The increase in abdominal visceral fat was marginally associated with the increase in cholesterol among A allele homozygotes $(r=0.67, p=0.07)$. Among the 24 subjects, the changes in abdominal visceral fat correlated negatively with post-overfeeding IGF1 concentrations $(r=-0.42, p=0.039)$. The number of carriers for the rare allele of the IGFBP3 Nde I $(n=4)$ polymorphism was low and no differences in response to overfeeding were found between genotypes. 
Table 2. Effects of overfeeding on blood levels of hormones in male twins in relation to the IGF2 APA I and IGFBP1 BGL II genotypes

\begin{tabular}{|c|c|c|c|c|}
\hline & \multicolumn{2}{|c|}{$I G F 2$ APA I genotypes } & \multicolumn{2}{|c|}{ IGFBP1 BGL II genotypes } \\
\hline & $\mathrm{AA} / \mathrm{AG}(n=12)$ & $\mathrm{GG}(n=12)$ & GG/GA $(n=16)$ & $\mathrm{AA}(n=8)$ \\
\hline \multicolumn{5}{|c|}{ Leptin (ng/ml) } \\
\hline After & $2.8(0.6)$ & $7.0(1.8) *$ & $5.0(1.4)$ & $4.7(1.0)$ \\
\hline Change & $2.1(0.5)$ & $5.4(1.3)^{*}$ & $3.8(1.1)$ & $3.7(0.9)$ \\
\hline \multicolumn{5}{|c|}{ Plasma insulin (pmol/l) } \\
\hline Change & $2.0(7.6)$ & $29.9(9.7)^{*}$ & $16.4(9.5)$ & $15.1(7.0)$ \\
\hline \multicolumn{5}{|c|}{ OGTT insulin area $\left(10^{3} \mathrm{pmol} / \mathrm{l} \cdot \mathrm{min}\right)$} \\
\hline Before & $50.2(5.8)$ & $58.8(5.8)$ & $56.1(5.1)$ & $51.2(7.3)$ \\
\hline After & $54.5(8.6)$ & $86.6(8.6) * *$ & $66.3(8.4)$ & $79.1(11.9)$ \\
\hline Change & $4.3(5.2)$ & $27.8(5.2)^{* * *}$ & $10.2(5.0)$ & $27.9(7.1)^{*}$ \\
\hline \multicolumn{5}{|c|}{ Plasma glucagon (ng/l) } \\
\hline Before & $661.9(27.6)$ & $614.4(33.5)$ & $619.6(26.3)$ & $675.4(37.9)$ \\
\hline After & $800.1(37.8)$ & $640.0(34.8)^{* * *}$ & $717.7(39.0)$ & $724.6(49.5)$ \\
\hline Change & $138.2(33.2)$ & $25.6(19.2)^{* * *}$ & $98.2(27.0)$ & $49.2(38.6)$ \\
\hline \multicolumn{5}{|c|}{ Insulin like growth factor I (IU/l) } \\
\hline Before & $982.2(123.0)$ & $1020.7(88.0)$ & $1091.9(98.9)$ & $820.6(71.5) *$ \\
\hline After & $1087.3(155.0)$ & $950.4(78.2)$ & $1125.7(119.1)$ & $805.1(49.5) *$ \\
\hline Change & $105.1(74.8)$ & $-70.3(80.9)$ & $33.8(80.4)$ & $-15.5(63.1)$ \\
\hline \multicolumn{3}{|c|}{$\begin{array}{l}\text { Values are means }(\mathrm{SE}) . p \text { values based on } 24 \text { subjects consid- } \\
\text { ered as unrelated persons are as follows: } \\
* p \leq 0.05 \\
* * p<0.03\end{array}$} & $\begin{array}{l}\text { en genotype gro } \\
\text { enotype means } \\
\text { ows: * and ** NS }\end{array}$ & $\begin{array}{l}\text { ach pair are } \\
d * * p \leq 0.05\end{array}$ \\
\hline
\end{tabular}

\section{Discussion}

The data of this study suggest that although the 189/ 189 bp genotype of the IGF1 (CT) n polymorphism has higher IGF1 concentrations, it is not associated with the responsiveness to the caloric surplus. However, IGF2 Apa I G allele homozygotes showed greater increases in OGTT insulin area, fasting insulin, leptin and subcutaneous fat concentrations than the A allele carrier in response to long-term overfeeding. Fat mass and amount of subcutaneous fat were lower before and after the overfeeding protocol in A allele carriers than in the homozygous subjects with the $\mathrm{G}$ allele. These observations correspond with the recently reported association of the IGF2 Apa I AA genotype with a lower mean body weight in a Caucasian population [7]. In the latter study, the IGF2 Apa I AA genotype was also associated with a higher serum IGF2 concentration [7], which was not measured in our study.

The increases in fasting insulin and OGTT insulin area in the male twins homozygotes for the IGF2 Apa $\mathrm{G}$ allele remained statistically significant after adjustment for the change in fat mass. Because the OGTT glycaemic area did not change, the IGF2 Apa $I \mathrm{G}$ allele homozygotes probably had a decrease in in-

sulin sensitivity with overfeeding. We speculate that the IGF2 Apa I G allele homozygotes could have had a decrease in IGF2 concentration or activity after exposure to the caloric overload. The latter could lead to alterations in glucose transport and glucose utilization in skeletal muscle thereby increasing peripheral insulin resistance [19]. The IGF2 Apa I GG subjects also showed less increase in total testosterone concentrations compared with the AA $+\mathrm{AG}$ subjects. IGF1 has been shown to suppress sex-hormone binding protein production [20], a major determinant of free sex hormone concentrations. Therefore, the observed trend for an increase in IGF1 in the AA + AG subjects could at least partly explain their greater increase in testosterone concentrations.

In the present study, the IGFBPI Bgl II A allele homozygotes gained more abdominal visceral fat and showed greater increases in OGTT insulin area and total cholesterol with overfeeding than $G$ allele homozygotes or heterozygotes. In addition, IGF1 concentrations among the IGFBPI Bgl II A allele homozygotes were lower before and after overfeeding and decreased slightly in response to overfeeding. Thus the IGFBP1 Bgl II AA genotype could increase IGFBP1 concentrations resulting in a decrease in the plasma concentration or metabolic activity of IGF1. 

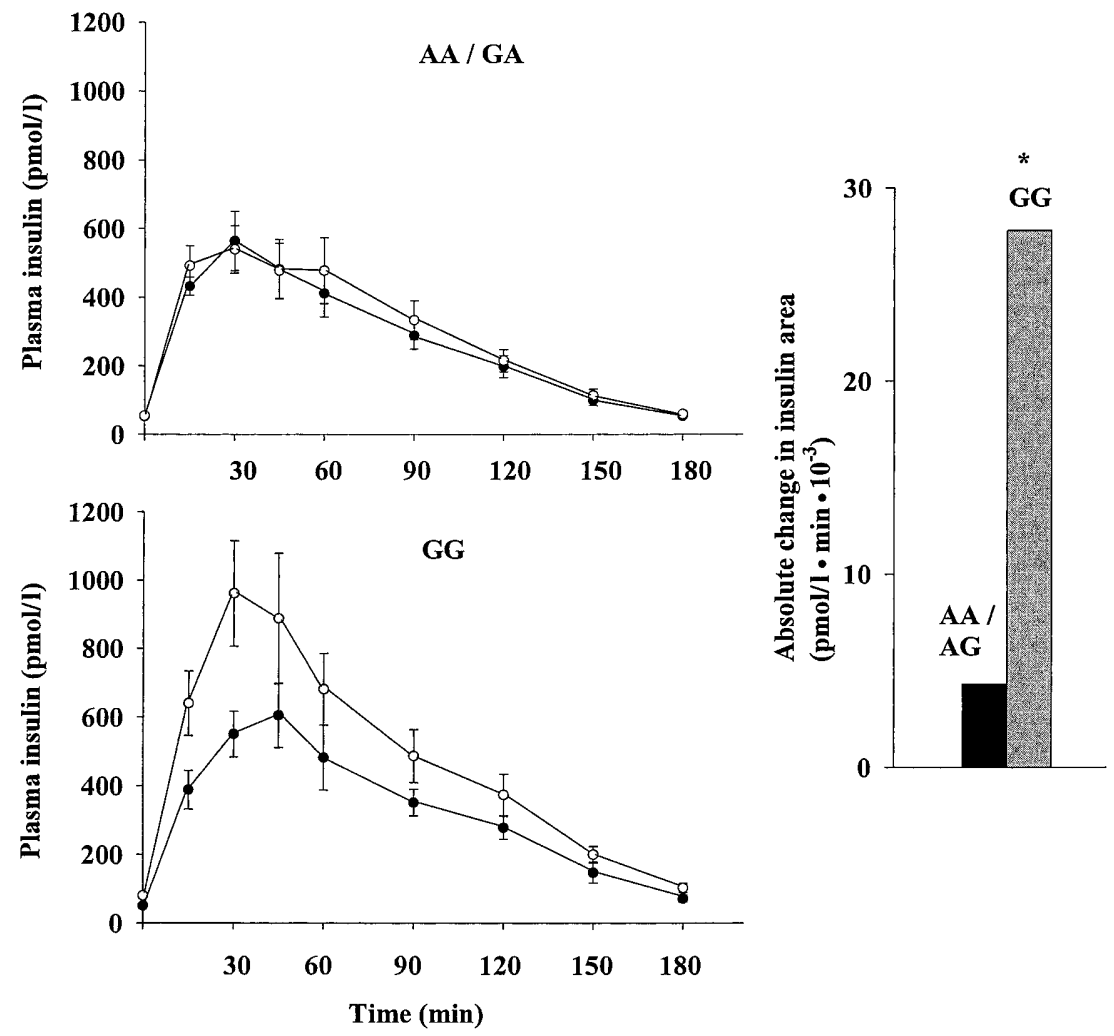

Fig. 1. Plasma insulin concentrations in the AA/GA and GG genotypes of the IGF2 Apa I polymorphism before (- - ) and after (- ) overfeeding as well as the absolute changes of the total insulin areas under the curve during the OGTT. $* p=0.004$ between the AA/AG and GG genotypes for the insulin area changes ( 24 cases) and $p=0.018$ when the mean of each pair $(n=12)$ is used

Low IGF1 concentrations in abdominal obesity have been reported previously [4]. In our study, the increase in abdominal visceral fat correlated negatively with post-overfeeding IGF1 concentrations. However, the hypothesis that IGFBP1 concentrations would be increased in homozygotes for the A allele does not agree with some studies showing lower IGFBPI concentrations in obesity [21]. The greater increase in the amount of abdominal visceral fat in the IGFBP1 Bgl II A allele homozygotes could explain some of the changes observed in OGTT insulin area and cholesterol. The latter hypothesis is supported by the notion that the increase in abdominal visceral fat correlated positively with the change in cholesterol among A allele homozygotes. Moreover, subjects who were IGFBP1 Bgl II A allele homozygotes had higher glucagon concentrations before and after overfeeding. The reason for the difference between A allele carriers and non-carriers is not known. Of note, IGF1 has inhibitory effects not only on the secretion of insulin but also on glucagon [3]. Therefore, the higher glucagon concentrations in AA subjects could be caused by the lower IGF1 concentration. Glucagon is also able to increase IGFBP1 concentration [20] and theoretically could change the fraction concentration of free circulating IGFs.

Both IGF2 Apa I and IGFBP1 Bgl II polymorphisms are located in non-coding regions and do not cause changes in the amino acid sequence of the IGF2 or IGFBP1 proteins. However, they could be in linkage disequilibrium with other mutations that have a more functional significance.

In summary, the IGF2 Apa I $G$ allele homozygotes had decreased insulin sensitivity independently of the increase in fatness in response to chronic overfeeding. The IGFBPI Bgl II A allele homozygotes had a greater accumulation of abdominal visceral fat which could have contributed to the overfeeding-induced early symptoms of the metabolic syndrome. Genetic variation at the IGF2 and IGFBPI loci could thus be among the factors responsible for the inter-individual differences observed in response to long-term alterations in energy balance and should be further investigated in large cohorts.

Acknowledgements. We are indebted to Drs. A. Tremblay and J-P. Després, and to J. Bouillon, S. Hamel, B. Zément, M. Lebrun, M. Marcotte, M. Chagnon, J. Lapointe, H. Bessette, G. Bouchard and S. Carbonneau for their contributions to this study. Gratitude is expressed to Dr. A. Nadeau and the staff of the Diabetes Research Unit for the glucose and insulin assays. We thank Dr. A. Belanger and his staff at the Molecular Endocrinology Laboratory for the steroid assays. Special thanks to G. Fournier and Dr. G. Thériault for their role in the management of the study. Thanks also to C. Leblanc for his statistical support. Supported in part by a grant (DK 
34624) from the National Institutes of Health and the Finnish Heart and Paavo Nurmi Foundations. C. Bouchard is partially funded by the George A. Bray Chair in Nutrition.

\section{References}

1. Baxter RC (1988) The insulin-like growth factors and their binding proteins. Comp Biochem Physiol B 91: 229-235

2. Hwa V, Oh Y, Rosenfeld RG (1999) The insulin-Like Growth Factor-Binding Protein (IGFBP) Superfamily. Endocr Rev 20: 761-787

3. Hussain MA, Schmitz O, Mengel A et al. (1993) Insulinlike growth factor I stimulates lipid oxidation, reduces protein oxidation, and enhances insulin sensitivity in humans. $\mathbf{J}$ Clin Invest 92: 2249-2256

4. Mårin P, Kvist $\mathrm{H}$, Lindstedt $\mathrm{G}$, Sjöström L, Björntorp $\mathrm{P}$ (1993) Low concentrations of insulin-like growth factor-I in abdominal obesity. Int J Obes Relat Metab Disord 17: 83-89

5. Rasmussen MH, Frystyk J, Andersen T, Breum L, Christiansen JS, Hilsted J (1994) The impact of obesity, fat distribution, and energy restriction on insulin-like growth factor1 (IGF-1), IGF-binding protein-3, insulin, and growth hormone. Metabolism 43: 315-319

6. Sun G, Cagnon J, Chagnon YC et al. (1999) Association and linkage between an insulin-like factor-1 gene polymorphism and fat free mass in the HERITAGE Family Study. Int J Obes Relat Metab Disord 23: 929-935

7. O'Dell SD, Miller GJ, Cooper JA et al. (1997) ApaI polymorphism in insulin-like growth factor II (IGF2) gene and weight in middle-aged males. Int J Obes Relat Metab Disord 21: 822-825

8. Bouchard C, Tremblay A, Després J-P et al. (1990) The response to long-term overfeeding in identical twins. N Engl J Med 322: 1477-1482

9. Behnke AR, Wilmore JH (1974) Evaluation and regulation of body build and composition. Prentice-Hall, Englewood Cliffs, pp 20-37

10. Siri WE (1956) The gross composition of the body. In: Lawrence JH, Tobias CA (eds) Advances in biological and medical physics, vol 4. Academic Press, New York, pp 239-280

11. Sjöström L, Kvist H, Cederblad A, Tylén U (1986) Detremination of total adipose tissue and body fat in women by computed tomography, ${ }^{40} \mathrm{~K}$, and tritium. Am J Physiol 250: E736-E745

12. Richterich R, Dauwalder H (1971) Determination of plasma glucose concentration by the hexokinase-glucose-6phosphate-dehydrogenase method (in German). Schweiz Med Wochenschr 101: 615-618

13. Nadeau A, Morissette J (1973) A new simple method for the linear transformation of the standard curve in insulin radioimmunoassay (in French). Union Med Can 102: 566-569

14. Unger RH, Eisentraut A (1968) Glucagon. In; Gray C (ed) Hormones in blood, vol 1, 2nd edn.). Academic Press, New York, pp 83-128

15. Bélanger A, Brochu M, Cliché J (1986) Plasma levels of steroid glucuronides in prepubertal, adult and elderly men. J Steroid Biochem: 24: 1069-1072

16. Neitzel H (1986) A routine method for the establishment of permanent growing lymphoblastoid cell lines. Hum Genet 73: 320-326

17. Tadokoro K, Fujii H, Inoue T, Yamada M (1991) Polymerase chain reaction (PCR) for detection of Apa I polymorphism at the insulin like growth factor II gene (IGF2). Nucleic Acids Res 19: 6967

18. Sun G, Chagnon M, Bouchard C (2000) A common polymorphism in the human insulin-like growth factor binding protein 3 gene. Mol Cell Probes 14: 55-56

19. O'Dell SD, Day INM (1998) Insulin-like growth factor II (IGF-II). Int J Biochem Cell Biol 30: 767-771

20. McCarty MF (1996) Up-regulation of IGF binding protein1 as an anticarcinogenic strategy: relevance to caloric restriction, exercise, and insulin sensitivity. Med Hypotheses 48: 297-308

21. Scacchi M, Pincelli AI, Cavagnini F (1999) Growth hormone in obesity. Int $\mathbf{J}$ Obes Relat Metab Disord 23: 260-271 